\title{
Automated Image Processing to Diagnose Exudates from Images of Retina
}

\author{
Sanjeevani Choudhary \\ Student \\ ITM University, Gurgaon
}

\author{
Jyotika Pruthi \\ Assistant Professor \\ ITM University, Gurgaon
}

\begin{abstract}
Diabetic Retinopathy (DR) is a significant reason for blindness. Exudates are one of the essential indications of diabetic retinopathy which is a fundamental driver of visual weakening that could be counteracted with an early screening procedure. In this approach, the procedure and learning of automated image processing to diagnose exudates from image set of retina are implemented.

The segmented image along with Optic Disk (OD) is selected. To Classify these segmented area, features considered as color base and texture are removed. The chosen feature vector is then grouped into exudates and non-exudates utilizing a Support Vector Machine (SVM) Classifier. An automated strategy to distinguish and restrict the vicinity of exudates from low-contrast digital images of Retinopathy patient's with non- dilated pupils is proposed.
\end{abstract}

\section{Keywords}

Diabetic Retinopathy, Exudate, Digital Image Processing, Optic Disc, Support Vector Machine (SVM) Classifier

\section{INTRODUCTION}

Diabetic Retinopathy is the normal retinal inconvenience connected with diabetes. It is a notable reason for blindness in middle and old age groups [1]. The evaluated pervasiveness of diabetes for all age groups globally was $2.8 \%$ in 2000 and $4.4 \%$ in 2030 implying that the aggregate number of diabetes patients is estimated to hike from 171 million in 2000 to 366 million in 2030 [2]. The International Diabetes Federation reports that more than 50 million individuals in India have this disorder and it is increasing rapidly [3]. Thus consistent screening is the most productive method for decreasing the vision trouble.

Diabetic Retinopathy is mostly created by the changes in the blood veins of the retina because of expanded blood glucose level. Exudates are yellow-white sores with moderately different edges. Exudates are proteins and lipids that stores and holes from the harmed veins inside the retina. Exudates are one of the essential indications of Diabetic Retinopathy [5]. Finding of Exudates by ophthalmologists is a difficult process as they need to invest a lot of time in manual examination and determination. Besides, manual recognition obliges utilizing chemical dilation material which requires significant investment and has negative reactions on patients. Subsequently automated screening strategies for exudates recognition have incredible centrality in economical expenses, time and work although to avoid the warning signs on patients.

Digital Color fundus images are generally utilized by ophthalmologists for diagnosing Diabetic Retinopathy. DR likewise causes various variations from the norm like neovascularisation, hemorrhages, microaneurysm, cotton fleece spots, and in later stages, retinal separation.

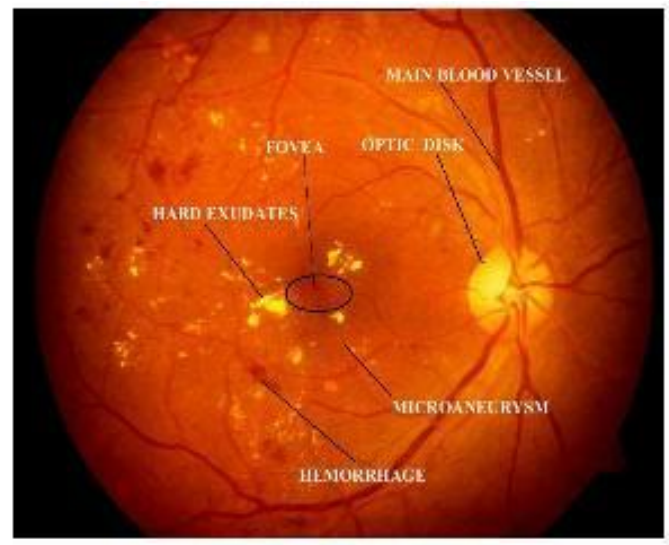

Fig.1: Diabetic Retinopathy image with various typical components.

Fig.1 outlines a typical retinal image marked with different peculiarity segments of Diabetic Retinopathy. Micro aneurysm is little secular pouches and shows as little red spots. This may prompt enormous blood clumps called haemorrhages. Macula is the middle part of the retina and has photoreceptors known as cons that are exceptionally sensitive to color and in charge of seeing fine subtle elements. The bright circular area from where the veins exude is called optic disk (OD). It is arranged at the back post temporal to the optic disk. The fovea characterizes the core of the macula and is the locale of visual sharpness.

\section{LITERATURE REVIEW}

Alireza Osareh et al [4] proposed a system for automated detection of exudates in view of computational Intelligence procedure the color retinal images were divided utilizing Fuzzy C-Means clustering vector were extricated and arranged utilizing support vector machine classifier.

Akara Sopharak et al [5] reported the consequence of an automated identification of exudates from low contrast digital images of retinopathy patients with non-expanded pupils by Fuzzy C-Means clustering. The identified result was accepted with master ophthalmologist's hand drawn ground truths. Four features, for example, force, hue, standard deviation on power and various edge pixels were separated and connected as input to coarse division utilizing FCM clustering system. Specificity, Affectability, positive prescient value (PPV), positive probability proportion (PPP) and exactness were utilized to assess the general execution of the framework.

Niemeijer et al [6] recognized the bright sore like exudates, drusen from colored retinal images and cotton fleece spots. In the first step, pixels were organized, fetching about a possibility outline incorporated the probability of every pixel to be a piece of a bright lesion. At that point, pixels with high probability were assembled into plausible lesion pixel 
clusters. In light of cluster attributes, every cluster was appointed a possibility demonstrating the likelihood that the cluster was a genuine bright sore. Sensitivities and specificities of the annotations on the 300 images by the automated framework were acquired. At last these clusters were delegated as exudates, cotton fleece spots or drusen.

Akara sopharak et al [7] proposed a progression of investigations on feature determination and exudates characterization utilizing naive bayes and Support Vector Machine (SVM) Classifiers. Firstly, they utilized naive bayes model to a training set comprising of 15 features removed from positive and negative samples of exudates pixels. Afterward, to get the best SVM, they utilized the best feature set of naive bayes classifier and consistently added the evacuated features to the classifier. For every composite of features, they completed a network search to determine the best combination of hyper parameters like resistance for training error and radial basis capacity width. They demonstrated that the naive bayes and SVM classifiers executed better than the NN classifier. They analyzed the best naive bayes and SVM classifier to a Nearest Neighbor classifier.

Walter et al [8] distinguished exudates from green channel of the retinal images as per their gray level variety. The exudates form was resolved utilizing numerical morphology methods. This system utilized three parameters: two limit values and size of the adjacent window. Exudates spots were at first discovered utilizing first limit value. The second limit states to the base quality, by which a participant pixel must vary from its including basis to be classified exudates. The author accomplished affectability of $92.8 \%$ and presciently of $92.4 \%$ against a set of 15 anomalous retinal images. However the author omitted a few sorts of errors on the border of the segmented exudates in their reported presentations and did not separate exudates from cotton fleece spots.

Sinthanayothin et al [9] reported the consequence of an automated identification of Diabetic Retinopathy by Recursive Region Growing systems on a 10X10 window utilizing chosen limit values. In the preprocessing steps, versatile, adjacent, contrast upgrade is implemented. The author reported an affectability of $88.5 \%$ and specificity of $99.7 \%$ for the recognition of exudates against a small dataset involving 21 unusual and 9 typical retinal images.

Phillips et al [10] distinguished the exudates by utilizing Global and local limit process. The input images were preprocessed to take out photographic non-consistencies and the contrast of the exudates was then improved. The lesion based affectability of this strategy was accounted between $61 \%$ and $100 \%$ considering 14 images. A disadvantage of this strategy was that other bright lesions, (for example, cotton fleece spots) could be recognized incorrectly.

\section{PROPOSED SOLUTIONS}

\subsection{Preprocessing of images}

The image preprocessing methods consists of Fuzzy C-means clustering, Discrete Wavelet Transform, Adaptive Histogram Equalization, Matched Filter Response and Gray scale Conversion for segmentation of blood vessels. The method we are working with is Gray scale conversion. The obtained image resolution is $1280 \times 1024$ in 24bit JPEG form.

Gray scale conversion: The color image of an eye is taken as input image and is changed over to a gray scale image. A typical procedure is to match the luminance of the gray scale image to the luminance of the color image. To change over any color to a gray scale representation of its luminance, initial one must get the estimations of its red, green, and blue (RGB) primaries in direct intensity encoding, by gamma extension. At that point, $61 \%$ of the green value, $28 \%$ of the red value, and $11 \%$ of the blue value is included. Hues in a image may be changed over to a shade of gray by assuming the effective brightness or luminance of the color and utilizing this quality to make a shade of gray that matches the required brightness. The powerful luminance of a pixel is determined utilizing the equation, $\mathrm{Y}=0.28 \mathrm{Red}+0.61 \mathrm{Green}+0.11 \mathrm{Blue}$. This luminance quality can then be transformed into a gray scale pixel. A gray scale digital image is an image in which the estimation of every pixel is a single example, that is, it conveys just intensity data. The Original DR influenced Eye Images are demonstrated in Fig. 2 beneath. The results of eye images after applying gray scale change are demonstrated in Fig.3 (a) $\neg$ (c) underneath.

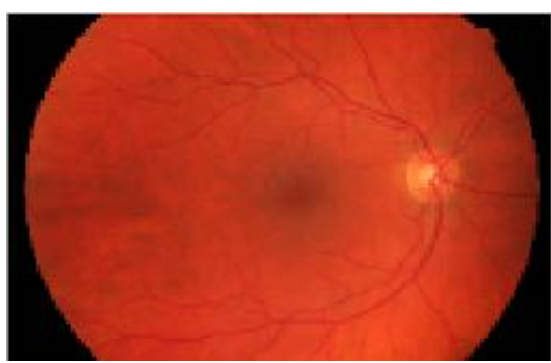

Fig. 2: Original Color image of eye

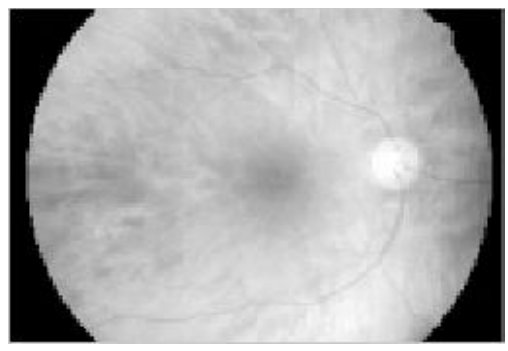

Fig. 3 (a): Original Red image of eye

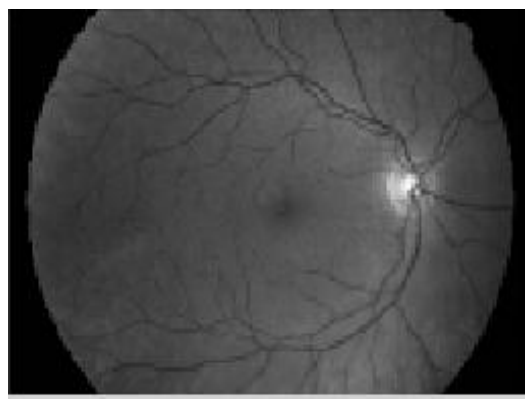

Fig. 3 (b): Original Green image of eye

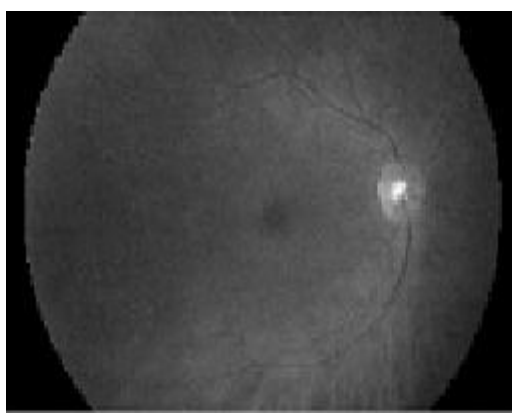

Fig. 3 (c): Original Blue image of eye 


\subsection{Feature Extraction and Exudates Detection}

To classify the confined segmented image into exudates and Non-exudates, various features in view of texture and color are divided utilizing Gray Level Co-event Matrix (GLCM). GLCM is a classification of how frequently distinctive combination of pixel brightness qualities occur in a pixel combination in a image. Every component $(i, j)$ in GLCM determines the quantity of times that the pixel with value $\mathrm{i}$ occurred evenly neighboring a pixel with quality $\mathrm{j}$. The subsequent matrix was examined and in view of the current data, the feature vectors are designed [11]. In view of texture, the extracted features are following:

i. Area of on pixels: It is the region of the white pixels with quality 1 on the high contrast image.

ii. Median: The median is the mathematical average of a set of values, or dispersion. Here in the eye image, it is developed by including the greater part of the pixel values together, then separating by the quantity of original values.

iii. Standard Deviation: The Standard deviation for a image is created by squaring every pixel values of all the individual examples, and then calculating average for the quantity of tests, $\mathrm{N}$. The standard deviation measures the spread of the information about the mean value. The example standard deviation, signified by s and follows:

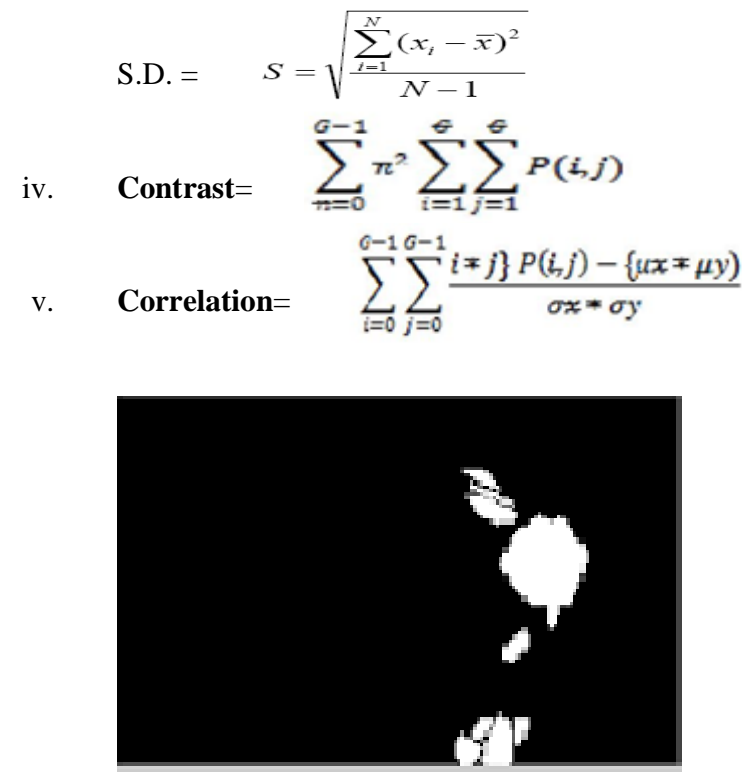

Fig. 4 (a): Exudate detection

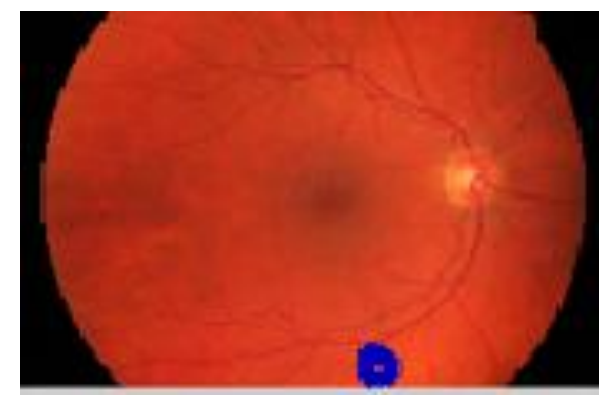

Fig. 4 (b): Original color image with optic nerve outlined
For the Detection of Diabetic Maculopathy, the Green Component is removed from the color fundus images. It is then preprocessed utilizing the median filter and convex hall segmentation. By applying these conversions, macula, which is the highlighted area of a image, is distinguished. If the exudates are exist in the macula, and then it demonstrates the vicinity of Diabetic Maculopathy as indicated by 4(a). Image outlined on the retina is transmitted to brain by optic nerve as demonstrated in figure 4(b). Optic disk is brighter than any piece of the retina image and is commonly round in shape.

\section{RESULTS}

In this methodology, we have explored and proposed a technique to automatically concentrate exudates from Diabetic Retinopathy images. The preprocessed colored retinal image is segmented into RGB colored images. The median filtering containing Optic Disk is utilized to choose and concentrate the features. The segmented colored image with Optic Disk is indicated in Fig. 5.a.

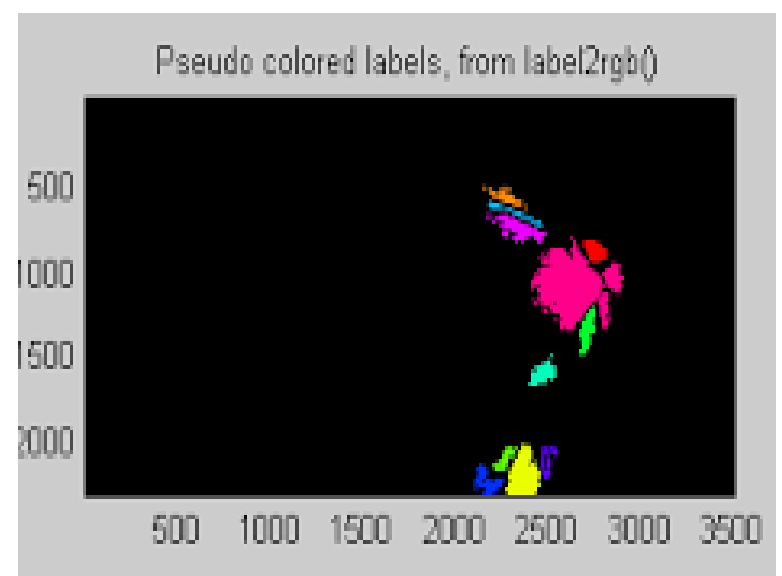

Fig 5(a): Segmented color image with Optic Disc

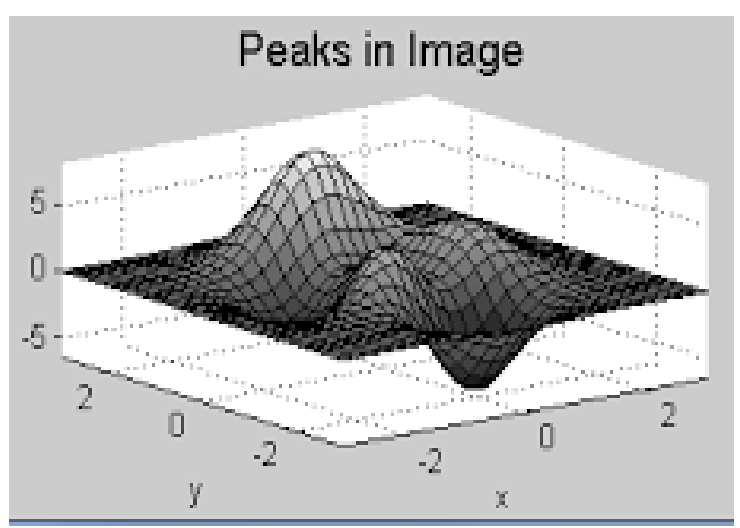

Fig 5(b): Success rate using classifier

In the chosen binary Green image, feature vector are removed utilizing Gray Level Co-event Matrix. With the assistance of these features, the chosen image is ordered into Normal (NonExudates) or Abnormal (Exudates) utilizing Support Vector Machine (SVM) Classifier. The achievement rate is discovered to be $96 \%$ shown by the figure 5 (b).

\section{CONCLUSION}

The proposed work in the paper identifies the existence of diabetic retinopathy generally in a patient by applying systems of digital image processing on fundus images. In this work, we have examined and proposed a programming $\neg$ based framework to distinguish normal case and diabetic retinopathy 
case. The Proposed framework showed a classification specificity of $100.00 \%$ and affectability of $99.45 \%$. This work demonstrates that support vector machines can be successfully utilized for image classification. Although by now lots of progress has been accomplished, there are as yet remaining problems and challenges for further research, for example, extracting more features and integration of classifiers and classification algorithms to give better execution and diminish the classification errors.

\section{ACKNOWLEDGMENTS}

Our thanks to the experts who have contributed towards development of the template

\section{REFERENCES}

[1] Olson. J. A, Strachana. F.M, Hipwell. J. H, "A comparative evaluation of digital imaging, retinal photography and optometrist examination in screening for diabetic retinopathy" Journal on Diabetes Med. Vol. 20, No. 7 .pp. 528- 534, July 2003.

[2] Alireza Osareh, Bita shadgar and Richard Markham,"A computational intelligence based approach for detection of exudates in Diabetic Retinopathy Images", IEEE Trans. onInformation Technology in Biomedicines, vol. 13, no. 4, pp. 535-545, July 2009.

[3] Saiprasad Ravishankar, Arpit Jain, Anurag Mittal, "Automated feature extraction for early detection of Diabetic Retinopathy in fundus images". IEEE Conference on Computer vision andpattern Recognition, pp. 210-217, August 2009.

[5] Akara Sopharak, Bunyarit Uyyanonvara, Sarah Barman, "Automatic Exudate Detection from Non-dilated Diabetic Retinopathy retinal images using Fuzzy C-
Means Clustering" Journal ofSensors, vol.9, No. 3, pp 2148- 2161, March 2009.

[6] Niemeijer. B.V, Ginnekan. S. R, Russell. M, and M. D. Abramoff, "Automated detection and differentiation of drusen, exudates and cotton- wool spots in digital color fundus photographs for diabetic retinopathy diagnosis", Journal on Investigate Ophthalmol. And Visual Science. vol. 48, No. 2 pp. 2260-2267, 2007.

[7] Akara Sopharak, Mathew N. Dailey, Bunyarit Uyyanonvara, Sarah Barman, Tom Williamson, Yin Aye Moe, "Machine Learning approach to automatic Exudates detection in retinal images from diabetic patients", Journal of Modern optics,Vol. 57, No. 2, pp. 124-135, Nov 2011.

[8] T. Walter, J.Klein, P.Massin and A.Erginary, "A Contribution of image processing to the diagnosis of Diabetic Retinopathy detection of exudates in color fundus images of the human retina", IEEE Trans. On Med. images, vol. 21, no. 10, pp. 1236-1243, 2002.

[9] C. Sinthanayothin, "Image analysis for automatic diagnosis of Diabetic Retinopathy", Journalof Medical Science, Vol. 35,No. 5, pp. 1491-1501, Jan 2011.

[10] Fleming. AD, Philips. S, Goatman. KA, Williams. GJ, Olson. JA, sharp. PF, "Automated detection of exudates for Diabetic Retinopathy Screening", Journal of Phys. Med. Bio., vol. 52, no. 24, pp. 7385-7396, 2007.

[11] Seongijin Park, Bohyoung Kim, Jeongjin Loe "GGO nodule volume preserving Non-rigid Lung Registration using GLCM texture analysis", IEEE Trans. On Biomedical Engg., vol. 58, no. 10, pp. 2885-2894, sept 2011 